\title{
Ouest Cameroun : valorisation des produits locaux. L'exemple du vin de raphia
}

Western Cameroon: valorization of local products. The case of raffia wine

\section{Célestin Kaffo, Nicole Doungtio Djeuta et Elat}

\section{(2) OpenEdition}

1 Journals

Édition électronique

URL : https://journals.openedition.org/economierurale/4003

DOI : $10.4000 /$ economierurale.4003

ISSN : 2105-2581

Éditeur

Société Française d'Économie Rurale (SFER)

Édition imprimée

Date de publication : 15 juillet 2013

Pagination : 61-79

ISSN : 0013-0559

\section{Référence électronique}

Célestin Kaffo, Nicole Doungtio Djeuta et Elat, « Ouest Cameroun : valorisation des produits locaux. L'exemple du vin de raphia », Économie rurale [En ligne], 336 | juillet-août 2013, mis en ligne le 15 juillet 2015, consulté le 28 juin 2022. URL : http://journals.openedition.org/economierurale/4003; DOI : https://doi.org/10.4000/economierurale.4003

(c) Tous droits réservés 


\title{
Ouest-Cameroun : valorisation des produits locaux L'exemple du vin de raphia
}

\author{
Célestin KAFFO • Université de Dschang, Cameroun \\ Nicole DOUNGTIO DJEUTA • Institut National de Cartographie, Yaoundé, Cameroun \\ ELAT • Institut National de Cartographie, Yaoundé, Cameroun
}

\begin{abstract}
La dépréciation de la valeur de la principale culture d'exportation et source de revenu qu'est le café depuis la fin des années 1980, a conduit les paysans à rechercher de nouvelles activités pour s'assurer un revenu quotidien. L'exploitation du palmier raphia participant de cet objectif s'est partout renforcée à travers la vente du vin de raphia. Ce commerce et la contribution des revenus qui en découlent dans l'amélioration des budgets familiaux ont fait croître le nombre de producteurs et d'intermédiaires commerciaux (parfois non producteurs) qui en tirent des revenus appréciables favorables à l'émergence d'une véritable " filière vin de raphia ». L'objectif de cette étude est d'analyser la filière vin de raphia en tant qu'exemple de mini-économie dans les ménages ruraux. Une lecture de cette évolution à Babadjou permet de mieux apprécier l'importance des produits locaux dans les stratégies de recomposition économique en cours dans les hautes terres de l'Ouest-Cameroun dans le nouveau contexte "post-café ".
\end{abstract}

MOTS-CLÉS : crise caféière, vin de raphia, économie locale, Ouest-Cameroun

\section{Western Cameroon: valorization of local products. The case of raffia wine}

The depreciation of the value of the main export crop and source of income that is coffee since the late 1980s has led famers to look for new activities to make a daily income. The exploitation of raffia palm participant of this objective is everywhere reinforce through the sale or raffia wine. The contribution of trade and income derived in improving family budgets have increased the number of producers and market intermediaries (sometimes non-producers) who derive substantial revenue favorable to the emergence of a real "raffia wine chain". The objective of this study is to analyze the "raffia wine chain" as an example of mini economy in rural households. An interpretation of this evolution in Babadjou is to better appreciate the importance of local products in the economic reconstruction strategies underway in the highlands of Western Cameroon in the new "post-coffee" context. (JEL: Q00)

KEYWORDS: coffee crisis, raffia wine, local economy, Western Cameroon

e café dès son introduction sur les hautes

terres de l'Ouest-Cameroun devient un « arbre à argent » donc synonyme de richesse. Mais depuis la fin des années 1980, soulignent Kuété et Uwizeyimana (2000), Nkankeu et Kaffo, (2012), à la suite de la chute des cours mondiaux qui a conduit à la déprise caféière et aux effets de la crise économique, cette paysannerie fait face à de profondes transformations socioéconomiques, spatiales et agriculturales. Progressivement, le café a cessé de jouer le rôle déterminant dans les revenus des ménages. L'impasse caféicole (déprise caféière) qui en découle a poussé les anciens producteurs de café Arabica et « cadets sociaux »- en majorité des migrants de retour vers les villages après la crise de la 
ville - à développer de nouvelles stratégies d'adaptation (Tsotezo, 2001).

$\mathrm{Au}$ sujet de cette impasse, Nkankeu et Kaffo (2012) démontrent qu'elle a été totale et irréversible, à tel point que l'État a procédé dans les années 1990 à la libéralisation des « joyaux du prince » et à la mise en place d'un cadre législatif visant à réglementer la recomposition spatio-économique et la poursuite (sans lui) du processus de développement en milieu rural. Les réponses paysannes se sont traduites, d'une part, par des reconversions massives à d'autres activités agricoles pourvoyeuses du numéraire au quotidien et, d'autre part, par de nombreuses tentatives d'organisation en OP (Organisations paysannes) et GIC (Groupes d'initiatives communes) afin de mieux prendre en main leur destin du moment où, du fait de la crise économique drastique et lancinante, l'État a démissionné de ses fonctions régaliennes d'encadrement du monde rural (Guillermou, Kamga, 2004 ; Guillermou, 2007 ; Fongang, 2008).

On comprend pourquoi Kuété et Uwizeyimana (2000) concluent dans leurs travaux que la campagne de l'Ouest est donc à la recherche de nouvelles marques, pour un nouveau départ, d'autant plus que ces sociétés ont maintes fois démontré leur capacité d'adaptation et leur aptitude à faire face à la conjoncture et à se reconstruire sur de nouvelles bases. Prioritairement, de nouveaux modes de production basés sur le vivrier et le maraîchage en particulier, furent rapidement inscrits parmi les réponses envisagées par les paysans et autres victimes de la déconfiture de l'économie que la culture d'exportation avait mise en place. Ces nouvelles spéculations sont devenues, sur le plan spatial, des concurrents sérieux au caféier et même une solution à la crise caféière (Ngapgue, 2007). Signe que le désarroi de la crise caféière fut de courte durée car les sociétés de montagne ont toujours fait preuve d'une grande flexibilité, du fait de conditions naturelles plutôt favorables (Uwizeyimana, 2009). La nouvelle dynamique agricole en court à l'Ouest-Cameroun est l'expression palpable de la remise en cause des anciennes formes d'organisation de l'espace, et donc une nouvelle construction territoriale : on semble désormais s'acheminer vers le triomphe de la petite exploitation familiale qui donne la priorité aux cultures vivrières aux dépens de la culture d'exportation. Le démantèlement des grandes plantations de café au profit des parcelles de cultures vivrières et maraîchères a abouti à une reconstruction des terroirs, voire à une nouvelle structuration de l'espace.

La kyrielle d'activités de substitution prouve à suffisance que dans le nouveau contexte «post-café », de nouvelles filières tentent de s'imposer en termes de viabilité et de rentabilité économique bien que ne suscitant pas un investissement durable de la part des agriculteurs. Outre le maraîchage et le vivrier marchand devenus ubiquistes à l'Ouest-Cameroun, la pluri-activité ou le petit élevage en expansion, l'exploitation du palmier raphia ${ }^{1}$ (Raphia vinifera) participant de cet objectif s'est renforcée. Bien que la raphiale n'occupait qu'une toute petite partie de la concession Bamiléké et son appropriation établie il y a plus de 50 ans, de nombreux caféiculteurs l'avaient adoptée comme plante d'ombrage. De nos jours, cette plante côtoie d'autres plantes dans le paysage agraire. Avec la chute des cours du café, le vin de raphia ${ }^{2}$ en termes de revenus est devenu le «nouveau café », un «marquage foncier », bref l'un des «symboles de la propriété individuelle de l'homme Babadjou », rôle jadis dévolu au caféier.

1. Il s'agit d'une plante à fonctions et usages multiples dans l'agroforesterie (Gautier, Fadani, 1994 ; Randrianjafy, Roger, 2005).

2. C'est une des variétés de boissons artisanales que l'on retrouve aussi bien au Cameroun que dans plusieurs autres pays tropicaux. Il existe entre autres le vin du palmier à huile, du palmier dattier. Cette boisson est très prisée par toute personne ayant séjourné à l'Ouest du Cameroun. 


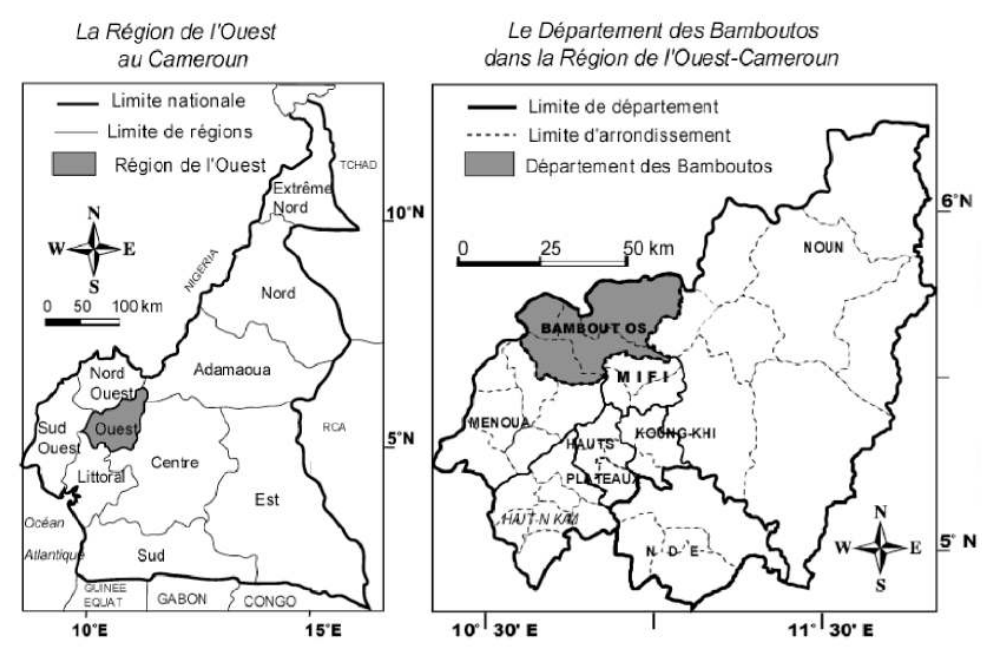

Le village Babadjou dans le Département des Bamboutos

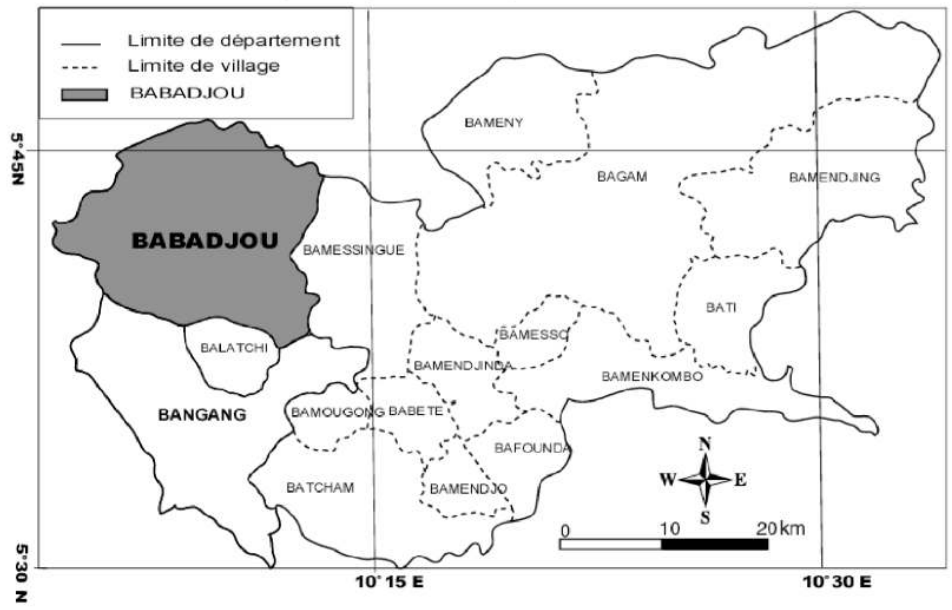

Source : Archives de I'INC, nov. 2012.

À la demande urbaine en nette croissante, le vin de raphia fait de plus en plus l'objet d'un marché particulièrement rémunérateur pour les producteurs et les revendeurs en même temps qu'il acquiert un rôle important dans le système de production de l'économie familiale essentiellement basée sur une agriculture de subsistance. L'engouement observé autour de sa production et son intégration dans le circuit commercial, bien qu'informel, traduit de fait d'après Oyono (1997), « le dynamisme des stratégies déployées par les paysans pour compenser la baisse des ressources monétaires en période de ruptures économiques ». À ce titre, Seignobos (2002) - soulignant l'intérêt socio-économique de l'exploitation de la bière de mil à Maroua dans les régions septentrionales du Cameroun - la présente comme étant le « rouage d'une économie informelle de survie nonobstant le procès en sorcellerie 


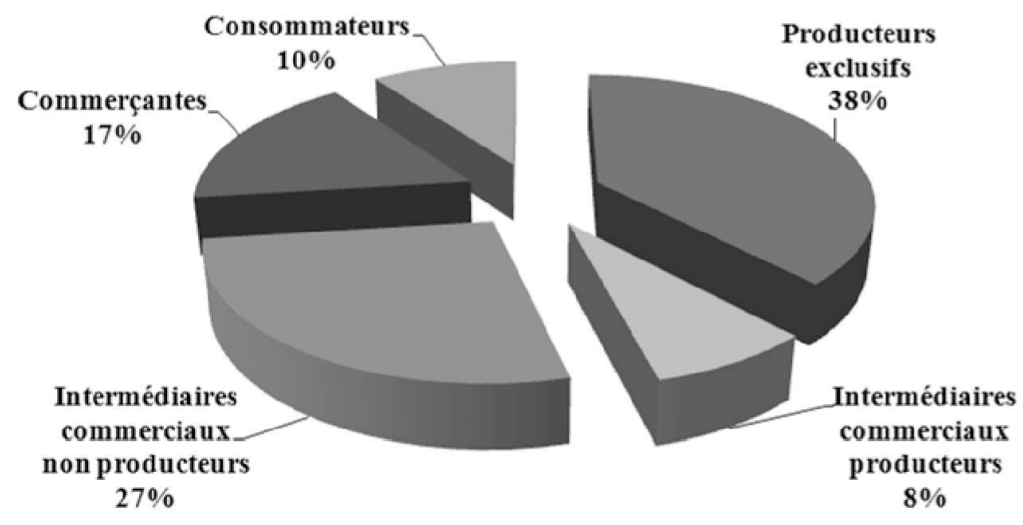

Source : I'auteur.

intenté à son endroit, sous couvert d'atteinte à la santé, à la morale et à l'environnement, pourtant elle participe à la fabrication d'une forme de citoyenneté ». Au demeurant, si la pratique d'une « activité de cueillette » est un héritage de la civilisation d'après Huetz De Lemps (2001), la conjoncture en constitue un atout pour sa valorisation. L'objectif de cette étude est d'analyser la filière vin de raphia en tant qu'exemple de mini-économie dans les ménages ruraux. Une lecture de cette évolution à Babadjou permet d'apprécier l'importance des produits locaux dans les processus de recomposition économique en cours dans les hautes terres de l'OuestCameroun causés par la déprise caféière.

\section{Cadre géographique et méthodologique}

Cette étude est effectuée dans la Région de l'Ouest du Cameroun et plus précisément dans le groupement Babadjou. Ce village (arrondissement éponyme né de la réforme administrative de septembre 1992) situé entre $5^{\circ} 38^{\prime}-5^{\circ} 48$ de latitude nord et $10^{\circ} 05^{\prime}-10^{\circ} 13$ de longitude est sur une superficie évaluée à $171 \mathrm{~km}^{2}$ fait partie des quatre arrondissements que compte le département des Bamboutos (figure 1). Babadjou comprend une vingtaine de quartiers. Appartenant à l'ensemble des hautes terres de l'Ouest, son relief est constitué de collines et de montagnes entrecoupées de vallées. Son climat est de type tropical humide avec une nuance d'altitude. Sa population est évaluée à 36929 habitants soit 5243 personnes résidentes en zone urbaine contre 31686 en zone rurale (Bucrep, 2005). D'après le rapport d'activité de la Délégation d'arrondissement de l'agriculture et du développement rural de Babadjou (Moffo, 2010), près de $80 \%$ des 14236 hommes qui résident en zone rurale tirent l'essentiel de leurs revenus après la fin du cycle caféier du vivrier marchand, du maraîchage, du petit élevage, du petit commerce, de l'artisanat et plus principalement de la vente du vin de raphia.

La méthodologie utilisée repose sur l'administration d'un questionnaire, des observations directes et des entretiens semi-directifs avec des personnes choisies au hasard. En l'absence de la liste exhaustive de tous les intervenants de la filière, nous nous sommes livrés à une observation à grande échelle. 
En fonction de leur implication dans la filière, ces enquêtés ont été classés par catégories. Ainsi, nous avons : des producteurs exclusifs, des intermédiaires (commerciaux producteurs ou non), des commerçantes ou buyam-sellams ${ }^{3}$ ou revendeuses de vin de raphia qui mènent leur activité dans les centres urbains environnants (figure 2) et des consommateurs finals.

Quant au support d'observation, six grappes correspondant aux principaux marchés de vente de vin de raphia à Babadjou (poste de péage de Matazem, Bachua, Kombou, Ndoji, Djinsso et Toumaka $)^{4}$ ont été choisies. Ces choix ont obéi à des critères essentiels : l'intensité de l'activité commerciale du vin de raphia par rapport à l'ensemble des points de vente sur toute l'étendue du village, l'ancienneté dans l'exploitation du raphia supérieure à 10 ans, la production et/ou la vente du vin de raphia comme activité principale, l'accessibilité aux points de vente et les motivations de la forte consommation du vin. Afin de parfaire notre analyse, les données collectées ont porté sur les mobiles réels de la frénésie vers le commerce du vin de raphia; les « gagnants » et les «perdants » d'une activité traditionnelle en effervescence ; les luttes réelles ou symboliques entre hommes et femmes, entre migrants de retour et anciens caféiculteurs ; la contribution des revenus tirés de la vente du vin de raphia dans le budget familial et sa capacité à constituer un appoint à l'économie locale ou à la lutte contre la pauvreté.

Face à la rareté des statistiques officielles sur ce type d'activité encore non imposable au niveau des producteurs, les chiffres utilisés dans la présente étude

3. Terme camerounais qui vient de l'anglais « buy and sell .

4. Ces marchés connus de tous les voyageurs ont l'avantage d'être situés sur la nationale $\mathrm{n}^{\circ} 6$ qui dessert les villes de Mbouda et Bafoussam dans la région de l'Ouest; de Santa et Bamenda dans la région du Nord-Ouest du Cameroun. sont issus des enquêtes parcellaires sur les principaux marchés identifiés. Malgré la disparité et le caractère diffus des circuits de distribution, ces statistiques permettent de montrer la montée en puissance de la filière vin de raphia.

\section{Les déterminants de l'intense exploitation du vin de raphia à Babadjou}

\section{La baisse des revenus des ménages ruraux}

La caféiculture a longtemps été le principal élément structurant de la société des hautes terres de l'Ouest-Cameroun. Son développement s'est accompagné de la mise en place d'un système coopératif qui, du fait de sa relative efficacité ${ }^{5}$ et de sa capacité à répondre à certains besoins sociaux, a suscité une adhésion massive et volontaire de la paysannerie. La diffusion d'une nouvelle logique économique impulsée par l'encadrement coopératif a rendu les paysans totalement dépendants et réfractaires à toutes stratégies compensatrices ou innovatrices en cas de crise (Nkankeu, Kaffo, 2012). Pour preuve, les revenus tirés de la culture d'exportation ont contribué tant à Babadjou que dans d'autres villages de l'Ouest-Cameroun, à la transformation et à la modernisation de l'habitat, à l'amélioration du cadre de vie, à l'appropriation de nombreuses épouses, aux paiements des frais de scolarité des enfants (Dongmo, 1981)... Cette contribution au progrès local s'est faite non seulement à travers des réalisations initiées par les populations mais aussi et surtout par les coopératives qui se sont engagées dans un important projet de développement régional intégré

5.L'action des Coopératives agricoles dans le développement rural portait sur la subvention des intrants, l'entretien des pistes de collecte de café, la création des écoles et centres de santé, l'achat de la totalité de la production caféière. 


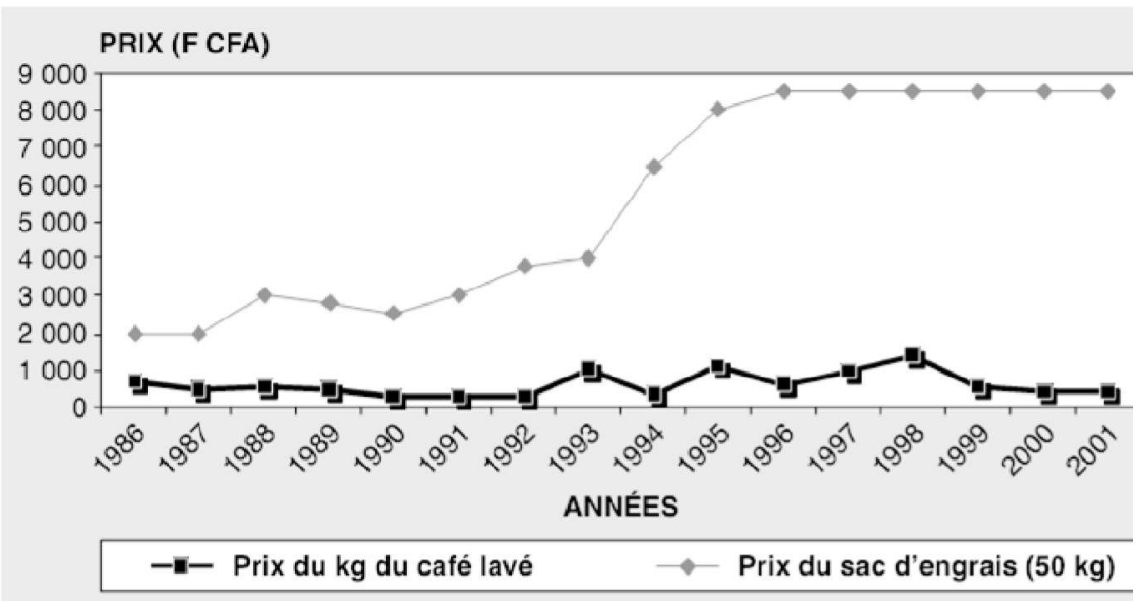

Source : Kaffo, 2005

par le cofinancement des activités du Projet de développement rural des plateaux de l'Ouest. Dans cette évolution, le vin de raphia et les produits des cultures vivrières avant la crise du café étaient destinés à la consommation. La vente d'une portion congrue n'apportait aux caféiculteurs que des revenus moins importants que ceux issus de la culture d'exportation.

Mais avec les crises caféière et économique des années 1980, les cours mondiaux du café ont périclité avec pour conséquence : la chute du prix du kilogramme du café payé aux planteurs (moins de 250 francs CFA/kg en 1990 contre 700 francs $\mathrm{CFA}^{6}$ en moyenne dans les années 1980), la hausse de $50 \%$ du prix des intrants agricoles et en somme, la baisse des revenus monétaires des paysans. La légère remontée du prix du kilogramme de la culture d'exportation (figure 3) observée à partir de 1993 (1 250 francs CFA) n'a été ni suffisante ni assez durable pour permettre d'envisager un renouvellement du capital arboré d'ailleurs totalement déraciné dans certains terroirs au profit

6. En francs CFA : 655,957 francs équivaut à $1 €$. du vivrier marchand et du maraîchage. La hausse de plus de $50 \%$ des prix des intrants agricoles libéralisés, vendus par la coopérative, a même plutôt renforcé la déprise. Lorsque le prix moyen d'un sac de $50 \mathrm{~kg}$ d'engrais minéral de «20-10-10», le plus utilisé à l'Ouest-Cameroun passe d'environ 2000 francs CFA en 1987 à plus 8000 francs CFA en $1995^{7}$, le taux de subvention des intrants (en pourcentage du prix de vente à l'exploitant) va connaître une baisse considérable, passant de $66 \%$ en 1987-1988 à environ $20 \%$ en 19901991, puis à $0 \%$ en 1992-1993. La dynamique des prix d'achat du kg de café et des engrais a eu un impact sur la caféiculture (Kaffo, 2005).

La dégradation consécutive des revenus en particulier et du niveau de vie en général conduira à un effort de diversification des activités productives, à la multiplication des sources de revenus et à la résurgence des activités complémentaires : petit élevage (volaille, chèvres), culture de haricots et de maïs, maraîchage, etc. (Kaffo, 1998,

7. De nos jours, le prix du même sac d'engrais minéral oscille entre 19000 et 22000 francs CFA. 
2005). Dans cette compensation, le raphia, en tant que plante pérenne, a presque remplacé le café au point de devenir un élément primordial, un «symbole de la propriété individuelle ». La vente du vin de raphia devient alors l'une des principales sources de revenu pour de nombreux ménages à Babadjou. L'engouement observé autour du vin de raphia montre que face à une paupérisation croissante, les populations ont souvent opposé des réactions sociales et surtout économiques qui se caractérisent par l'exploitation de produits locaux plus aptes à créer des revenus immédiats et des emplois, fussent-ils temporaires (directs ou indirects). À ce titre, les jeunes - « diplômés » ou déscolarisés -, sans emplois ou ayant perdu leur emploi en ville et de retour au village, ont - dans l'attente d'une activité plus rentable - su saisir cette nouvelle opportunité qui « rapporte » des revenus substantiels.

Comme autre facteur et non des moindres, la hausse du prix des boissons modernes a favorisé, à des proportions variables, l'ajustement dans la demande du vin de raphia en fonction des circonstances ${ }^{8}$. On comprend donc à la lecture de tout ce qui précède que le déclin des revenus participe du repli à l'exploitation et à la consommation des produits locaux.

\section{Des conditions et techniques}

L'engouement qu'ont les ruraux et les néoruraux dans l'exploitation du raphia émane des conditions de cueillette somme toute particulières (moins contraignantes surtout en temps contrairement au café et au maraîchage) bien que nécessitant des

\footnotetext{
8. Lors de grandes cérémonies, les populations rurales et urbaines par effet de mode consomment beaucoup plus les boissons modernes (bières, vin rouge ou autres liqueurs de valeur) que le vin de raphia lorsque la possibilité d'opérer un choix leur est offerte. Dans le cas contraire, elles festoient au vin de raphia.
}

efforts physiques. Les conclusions des travaux menées par Delarue (2007) en Guinée forestière rejoignent les pratiques et habitudes des producteurs rencontrés dans le cadre de cette étude. Elles montrent que le travail demeure le facteur de production le plus important. L'entretien de la raphiale se limite à l'élagage des stipes secs, à la transplantation des jeunes pousses dans les espaces les plus favorables à leur développement : cette transplantation est réalisée ponctuellement par les agriculteurs, lorsqu'ils ont du temps libre, avec un coût d'opportunité du travail nul par conséquent. La récolte du vin intervient lorsque l'arbre est sur le point d'émettre son premier bourgeon floral avant la fruitescence (lorsque l'arbre produit sa première fleur, sa sève n'est d'ailleurs plus exploitable). L'apex est incisé à sa base chaque jour au moyen d'une lancette ou d'un couteau de vigneron et la sève est recueillie dans une calebasse. Le matériel utilisé pour l'exploitation du vin de raphia se limite à quelques dames-jeannes et/ou des bidons en plastique, à une lancette, un plantoir, une machette, la gouttière, un matériel au coût tout à fait dérisoire et à durée indéterminée sauf en cas de perte.

L'exploitation d'un pied de raphia ne demande pas beaucoup de temps, mais de la régularité. La récolte du vin de raphia a lieu matin et soir. Les producteurs que nous avons interrogés se déclinent en trois catégories en fonction du nombre de pieds de raphia en cours d'exploitation :

- les petits producteurs : 5 à 10 pieds ;

- les producteurs moyens : 10 à 25 pieds ; - les grands producteurs : plus de 25 pieds.

Le temps de récolte est fonction du nombre de pieds à exploiter et de la distance à parcourir entre le domicile et le champ, et du champ au marché. Plus la distance est longue et plus les pieds à exploiter sont nombreux, le temps à y consacrer est considérable. Certains grands producteurs commencent la cueillette très 
tôt soit à partir de 3 heures du matin pour la récolte de la matinée et 16 heures pour celle du soir.

\section{La maîtrise des « savoirs locaux »}

L'analyse des données de terrain a révélé que la maîtrise des « savoirs locaux » de production et de cueillette (exploitation des plants matures, protection et entretien quotidiens de l'exutoire contre l'infiltration des eaux, les insectes et animaux sauvages tels que les civettes, les rats palmistes, etc.) joue un rôle de grande envergure dans la production du vin de qualité. Cette plus-value a, par certains égards, constitué un facteur de renforcement de la forte demande locale et urbaine du vin de raphia surtout de Babadjou. Les raphiales des vallées hydromorphes commencent à produire à partir de la $10^{\mathrm{e}}$ année tandis que la production de celles des versants non drainés doit attendre au moins la $15^{\mathrm{e}}$ année. Le respect de ces principes, gage de la production et de la mise sur le marché d'un vin de qualité, a toujours été le cheval de bataille de l'autorité traditionnelle qui interdit sous peine de sanctions ${ }^{9}$, la saignée d'un raphia immature $^{10}$. La longévité du cycle de production du palmier raphia dépend non seulement des différences d'ordre génétique, de l'influence de l'altitude, des influences pédologiques, hydrologiques et climatiques mais aussi du mode d'entretien de l'exutoire d'extraction. Il est possible de considérer qu'un pied de raphia peut, dans des conditions optimales, produire environ

\footnotetext{
9. Les sanctions sont variées : corvée à la chefferie, interdiction de vendre le vin sur les marchés du village ou la consommation du vin jugé de mauvaise qualité, peu importe la quantité.

10. Par ailleurs, la saignée d'un pied de raphia immature produit un vin de mauvaise qualité : fade à la récolte et aigre après fermentation. D'après les informations recueillies sur le terrain, le vin de mauvaise qualité se reconnaît par la présence, autour de la calebasse ou du récipient, des mouches en lieu et place des abeilles.
}

300 à 400 litres de vin de raphia entre 3 à 4 mois avant que l'arbre ne meure. Il faut souligner pour s'en convaincre que le choix du site d'exploitation (bas-fonds aux sols hydromorphes ou le tiers des versants non drainés) et la saison militent en faveur d'une production d'un vin de qualité. Le vin de saison humide est moins prisé que celui de saison sèche puisque le chargement de la nappe et des racines du palmier raphia altère la saveur du breuvage.

Sur le plan biologique, le vin de raphia est une boisson dont les qualités organoleptiques ne sont pas constantes dans le temps. Lorsqu'il vient d'être récolté, il présente une saveur sucrée avec un taux d'alcool égal à zéro. Ce vin doit être consommé soit immédiatement après récolte, soit dans un délai d'un à deux jours, après avoir été de préférence mélangé avec des récoltes plus récentes. Mais à plus de deux jours, il devient franchement acide et peut causer des brûlures d'estomac. Dès qu'il est embouteillé, la fermentation est rapide et provoque une acidification et une augmentation de la teneur en alcool avec un fort dégagement gazeux. Mais en l'absence de la vulgarisation des modes de conservation moderne en cours d'élaboration (Meutchieye, 2007), le producteur pour empêcher la fermentation rapide du vin qui le rend aigre - applique une mince couche d'huile de palme sur le bouchon du récipient ou y introduit quelques feuilles de Combretum micranthum. Cette technique est appropriée lorsque le vin doit être transporté sur une très longue distance ou être exposé à de fortes températures.

\section{Le raphia : symbole de la tradition des peuples des Grassfields}

Nonobstant la modernisation de la société traditionnelle et la prolifération des boissons modernes (bières et autres liqueurs), le vin de raphia reste l'un des symboles forts de la tradition des peuples des Grassfields. 
Autant il scelle l'alliance entre les familles lors des célébrations des mariages, conforte les liens entre les membres d'une réunion, permet de nouer un pacte ou de mettre fin à un litige (Gautier, Fadani, 1994), autant il joue un rôle dans la pharmacopée traditionnelle. En pays Bamiléké, le respect accordé au vin de raphia interdit à tout consommateur de boire en public en tenant son verre de vin de la main gauche. Un tel acte est considéré comme un manque de respect à la tradition dans tous ses aspects. Ainsi, le renforcement de l'exploitation du raphia tient au fait que le vin de raphia a longtemps été à la base des relations sociales et culturelles des peuples des Grassfields.

Autour d'une bouteille de vin de raphia dans les buvettes communément appelées matango club et «placées aux confluents des contraires ${ }^{11}$, se côtoient traditionalistes et modernistes, vieux et jeunes, débrouillards et fonctionnaires [...] tout le monde trouve ce qu'il y cherche : l'oubli du quotidien, un retour identitaire, mais aussi la construction de nouvelles connaissances. En somme, c'est là où l'on apprend beaucoup les nouvelles de la ville », déclare un consommateur (Seignobos, 2002). Le phénomène de cohabitation entre la vente du vin de raphia et de la boisson moderne est devenu ubiquiste dans certains débits de boisson moderne à Babadjou. Le caractère hautement social a enrichi le volet économique qui se développe et se maintient solidement à travers l'émergence de la filière vin de raphia.

\section{Organisation et acteurs}

Faut-il encore le rappeler, la déconfiture de la culture d'exportation a modifié l'orientation économique des systèmes de production de l'espace à l'Ouest du Cameroun

11. Expression empruntée à Seignobos (2002) pour signifier l'ambiance et l'hétérogénéité de la clientèle dans les buvettes de vin de raphia. au profit des produits susceptibles de tenir l'économie familiale. Ainsi, le vin de raphia y a revêtu un caractère spéculatif, offrant d'intéressantes perspectives d'accumulation selon le mode de commercialisation établi tout en se plaçant comme source de revenus. Le marché rural et urbain fait appel à différents types d'acteurs qui organisent des circuits de distribution complexes et variés avec d'une part les marchés et d'autre part les acteurs.

\section{Les marchés permanents}

Les principaux lieux permanents de vente du vin de raphia sont identifiables dans le village Babadjou (arrondissement éponyme). Ceux-ci se trouvent le long du grand axe routier bitumé où convergent quotidiennement environ $75 \%$ des agents de la filière et auxquels sont reliés de nombreux marchés de quartiers et de points de collecte (figure 4). Ces marchés constituent les principaux centres d'approvisionnement. Les marchés les plus réputés sont, par ordre d'importance :

Le marché de Ndoji (Bamedji) situé à $52 \mathrm{~km}$ de la ville de Bamenda et de Bafoussam. Il a la réputation d'être le plus important marché de vin de raphia à Babadjou après celui de Toumaka. C'est ici que se ravitaillent quotidiennement la majorité des «buyams sellams » de Mbouda et de Bafoussam et de bien d'autres particuliers locaux et urbains. Il est caractérisé par la qualité du vin produit ou livré. En tant que lieu de convergence des livreurs des quartiers intérieurs, plus de 10000 litres de vin de raphia y sont collectés et écoulés quotidiennement.

Le marché de Toumaka situé au sud du village. Il a la particularité d'abriter des agences de voyages à partir desquelles sont approvisionnées certaines grandes villes (Douala et Yaoundé).

Les marchés de Kombou, de Bachua et du poste de péage de Matazem situés au 


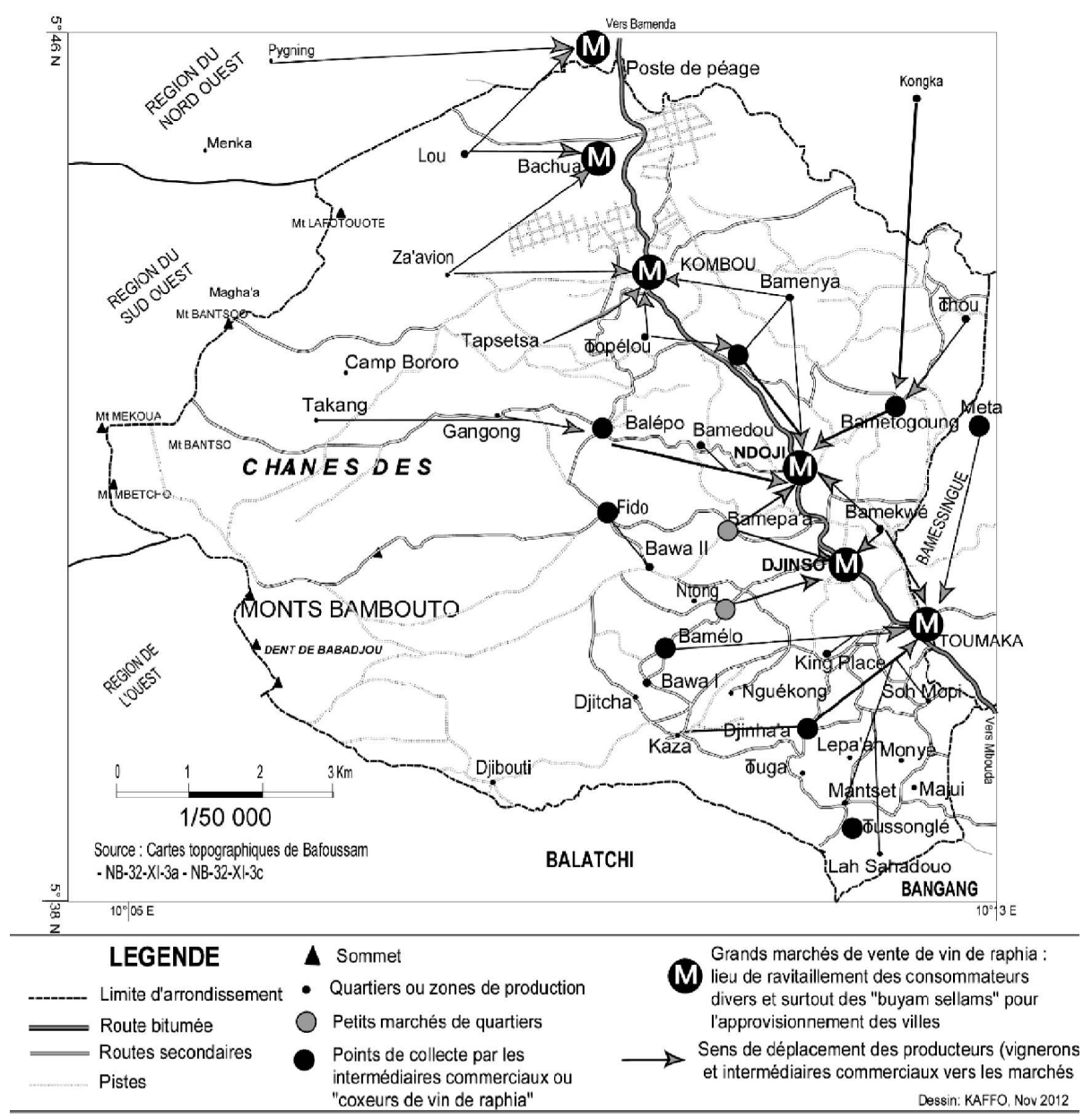

Source : Carte topographique de Bafoussam au 1/50 000 et enquête de terrain, 2012.

nord du village (la frontière du Cameroun anglophone). Ils desservent principalement les villes de Santa et de Bamenda. Mais aussi, ils servent de lieu d'approvisionnement de certaines revendeuses en provenance de Mbouda, de Bafoussam et de Douala.

Le marché de Djinso à cause de sa proximité avec celui de Toumaka est le moins important. L'affluence n'y était constatée comme dans d'autres marchés intérieurs, que les jours des marchés périodiques qui se tiennent sous huitaine. Mais avec l'entrée en scène des coxeurs (rabatteurs) de vin de raphia, l'activité y est devenue permanente.

\section{Les moyens de transport}

Les moyens de transport du vin de raphia des zones de production vers les points de collecte, les petits marchés de quartiers ou les marchés permanents de vente sont divers et ont connu une évolution au fil des ans au niveau local. Les moyens les plus 
utilisés étaient la tête d'homme, le vélo et la brouette. Mais avec les motos, le marché du vin de raphia a connu une plus-value d'autant plus qu'elles facilitent le transport et la production en grande quantité (200 litres environ) des zones reculées vers les principaux marchés ou points de collecte.

\section{Acteurs, circuits de distribution et de commercialisation}

\section{Principaux acteurs de la filière}

Les acteurs des différentes étapes de la filière vin de raphia sont les premières personnes qui peuvent avoir accès aux avantages ou subir les inconvénients de ce secteur. Il s'agit des producteurs ruraux, des intermédiaires commerciaux, des commerçantes et des consommateurs finals. Ces groupes d'acteurs sont loin d'être homogènes au regard des rôles et tâches respectifs.

Les producteurs ou vignerons. Ce sont les acteurs essentiels de la chaîne de commercialisation du vin de raphia. Ils se recrutent parmi les jeunes sortis du système éducatif, les anciens caféiculteurs, les retraités ; bref les ruraux et les néoruraux.

Les intermédiaires commerciaux. Ce sont en général des producteurs non propriétaires des raphiales ou des « vignerons à col blanc ». Généralement, ils servent de liaison entre les producteurs éloignés et d'autres acteurs de la filière. Ils achètent du vin de raphia qu'ils transportent à l'aide de leur moto et livrent en gros aux acheteurs des marchés périodiques, aux commerçantes ou à une clientèle garantie de la ville.

Les commerçantes ou « buyams sellams ». Ce sont généralement des femmes. En tant qu'actrices stratégiques du système, elles se subdivisent en deux sous-groupes : premièrement, celles qui achètent d'importantes quantités de vin de raphia dans les zones de production et les livrent à d'autres commerçantes en ville. Deuxièmement, les commerçantes-détaillantes (qui vendent personnellement le vin acheté).
Les consommateurs finals. D'origines diverses et variées, ils sont composés de ceux qui achètent soit pour leur consommation personnelle, soit pour assister des tiers à des manifestations diverses.

\section{Circuits de distribution et de commercia- lisation}

L'émergence de la filière vin de raphia met en exergue grossièrement deux volets distincts dans le circuit de vente et de distribution (figure 5) : le volet commercial reconnu et le volet traditionnel sans incidence financière. Ce dernier prend en compte les offres aux proches à la demande ou à l'occasion des manifestations socioculturelles (obsèques, funérailles, mariages, etc.). Se rapportant au volet commercial reconnu, on distingue trois types de circuits.

- Type 1 : producteurs/consommateurs. Ces circuits s'organisent de plusieurs manières : soit sur les marchés physiques locaux (des quartiers intérieurs ou des grands marchés) fréquentés par une myriade de producteurs, soit « bord raphiale » ou « bord champ » lorsque les acheteurs, collecteurs ou consommateurs, se rendent personnellement dans les raphiales pour effectuer achats et enlèvements. Le règlement des transactions se fait au comptant ou à crédit. Il s'agit d'une pratique parfois à risque car l'acheteur peut ne pas payer sa dette à la date convenue au producteur ou disparaître de la circulation s'il est non-résident.

- Type 2 : producteurs/commerçantes/ consommateurs. Dans ce circuit, le vin de raphia est acheminé aux consommateurs urbains par l'entremise des revendeuses qui l'achètent et le revendent aux consommateurs dans les bistrots.

- Type 3 : producteurs/intermédiaires commerciaux/commerçantes/consommateurs. Ce circuit fait intervenir, d'une part, un ensemble d'intermédiaires commerciaux propriétaires de motos et, d'autre part, des commerçantes (grossistes ou détaillantes). Ces intermédiaires propriétaires 


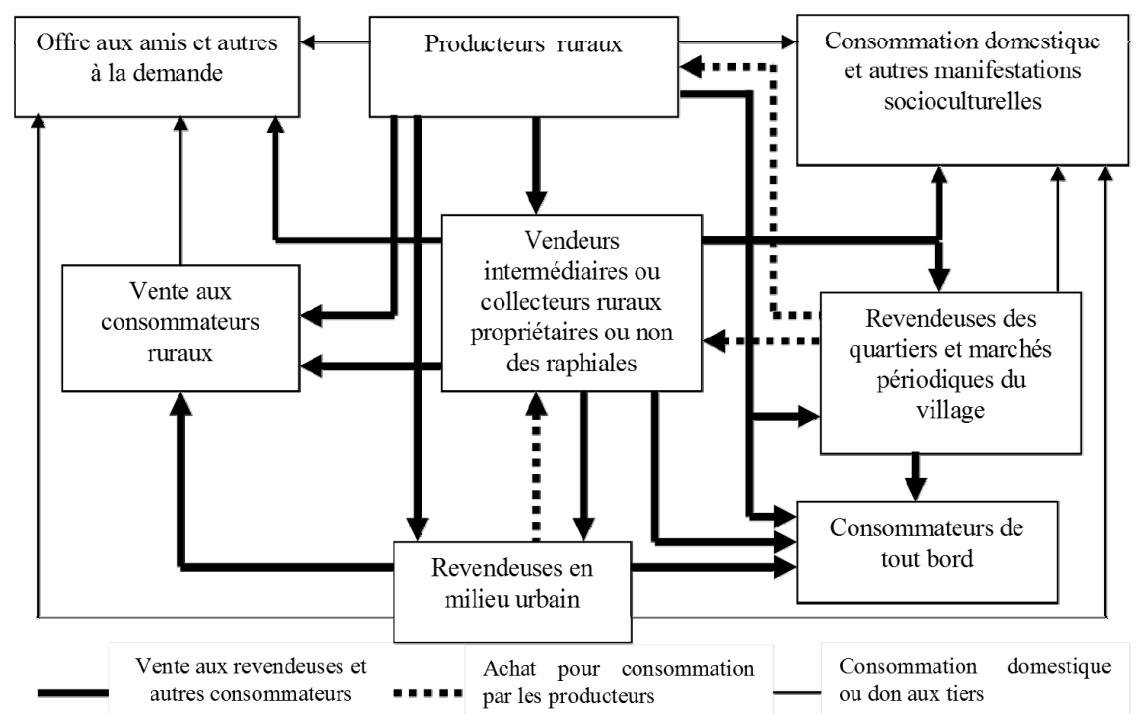

Source : Enquêtes et observations de terrain.

Figure 6. Polarisation de l'espace par les lieux d'échange et les centres de consommation du vin de raphia

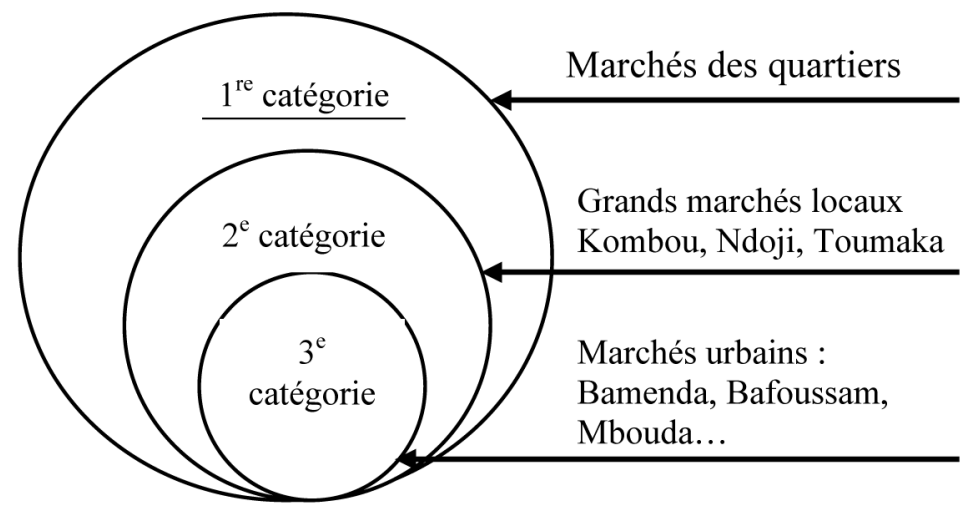

de motos ne sont pas généralement propriétaires de raphiales. Ce sont des coxeurs de vin de raphia.

Dans d'autres localités telles que Bafoussam et Dschang, certains producteurs s'érigent en vendeurs ambulants. Ils parcourent les artères des quartiers avec des bonbonnes et quelques bouteilles d'un litre dans les pousse-pousse soit dans une corbeille sur la tête soit sur un vélo à la recherche d'acheteurs potentiels.

En somme, le vin de raphia est généralement vendu par des acteurs opérant au quotidien sur des marchés divers. Lesdits marchés s'organisent autour du binôme vendeurs/acheteurs (consommateurs directs 
RECHERCHE

Célestin KAFFO, Nicole DOUNGTIO DJEUTA, ELAT

Tableau 1.Provenance des commerçantes et quantités moyennes achetées/jour dans trois principaux marchés de Babadjou

\begin{tabular}{l|c|c|c|c|c|c|c|c|c}
\multirow{2}{*}{ Destination } & \multicolumn{8}{c|}{ Marchés ou zone de dénombrement } \\
\cline { 2 - 13 } & \multicolumn{3}{|c|}{ Ndoji } & \multicolumn{3}{c|}{ Kombou } & \multicolumn{3}{c}{ Toumaka } \\
\cline { 2 - 12 } & $(1)$ & $(2)$ & $(3)$ & $(1)$ & $(2)$ & (3) & (1) & (2) & (3) \\
\hline Mbouda & 25 & 150 & 3000 & 15 & 100 & 2000 & 5 & 125 & 2500 \\
\hline Bafoussam & 20 & 300 & 6000 & 12 & 98 & 1840 & 5 & 50 & 1000 \\
\hline Douala & $/$ & $/$ & $/$ & $/$ & $/$ & $/$ & 10 & 200 & 4000 \\
\hline Bamenda & $/$ & $/$ & $/$ & 30 & 200 & 4000 & 2 & 80 & 160 \\
\hline Dschang & $/$ & $/$ & $/$ & 1 & 5 & 100 & $/$ & $/$ & $/$ \\
\hline Marché local & 30 & 200 & 4000 & 19 & 150 & 3000 & 5 & 10 & 200 \\
\hline Total & 75 & 650 & 13000 & 77 & 553 & 10940 & 27 & 465 & 7860
\end{tabular}

Note : (1) nombre de commerçantes, (2) : total des bonbonnes de 20 litres, (3) : quantités de vin en litre.

Source : Enquête de terrain réalisée du 2 au 5 novembre 2012.

ou revendeurs), généralement des revendeuses et, depuis un certain temps, autour de nombreux intermédiaires commerciaux (coxeurs) ayant intégré le circuit. Cette situation est identique à celle des produits forestiers non ligneux où les intermédiaires commerciaux font subir une transformation plus ou moins poussée.

\section{Polarisation de l'espace par les lieux d'échange et centres de consommation}

Le rôle polarisateur d'un marché, selon Dongmo (1976), peut se mesurer par l'origine de ses vendeurs et de ses acheteurs : cela permet de voir la région qui dessert le marché considéré. Sur la base dudit critère, les marchés de vin de raphia de Babadjou forment un vaste réseau hiérarchisé réparti en trois catégories suivant leur taille, leur couverture spatiale et géographique (figure 6).

La première catégorie, la plus vaste, regroupe les marchés intérieurs de petite à moyenne taille localisés dans les quartiers, spécialisés dans la vente dans les buvettes et l'approvisionnement des gros marchés locaux. Ils servent de points de ravitaillement des consommateurs, revendeurs ruraux et de plus en plus des intermédiaires commerciaux.

La deuxième catégorie couvre les grands marchés locaux, de véritables pôles de collecte autour desquels, s' organise quotidiennement un commerce intense entre 6 heures et 10 heures D'où partent d'énormes quantités de vin de raphia pour l'approvisionnement des différents centres de consommation comme l'atteste le tableau 1.

Les données présentées dans le tableau ci-dessus donnent un aperçu des quantités moyennes écoulées quotidiennement (environ 31810 litres). Il est difficile, à cause du caractère diffus du circuit de distribution et de la complexité des intervenants, d'appréhender la production totale par marché et par jour.

La troisième catégorie est constituée des grands marchés urbains tels que Mbouda, Bafoussam, Bamenda, Douala, Yaoundé... Ici, le marché est tenu par les femmes buyam-sellams. Ces commerçantes, bien qu'exposées à la concurrence comme dans toute activité informelle, tirent profit des écarts spatio-temporels de prix plus importants que les producteurs ruraux. 


\section{Des prix et des conditions de vente}

Le marché de vin de raphia à Babadjou est extrêmement variable : son rôle dans le regroupement et la distribution du produit peut changer considérablement au cours d'une même journée et d'un jour à l'autre. Malgré les variations intra- et inter-journalières, voire intra-annuelles de l'offre, de la demande et des prix pratiqués, le pouvoir de négociation des commerçants et des producteurs dépend du type de contrat, de la proximité sociale ou l'affinité identitaire, spatiale et économique. Le marché de vin de raphia intègre les relations sociales dans les transactions.

Au sujet du contrat, deux types ont été identifiés : le « gré à gré » ou vente libre et le mode « client» où le producteur et la revendeuse ou encore le coxeur sur la base d'un contrat verbal, s'accordent sur le prix d'achat indépendamment de son évolution sur le marché. Chacun des deux systèmes présente des forces et faiblesses au regard de l'évolution et de la variation des prix selon les saisons et certains grands événements socioculturels. D’une manière générale, les prix connaissent d'incessantes variations au gré des saisons. D'octobre à mars (saison sèche et des funérailles en pays Bamiléké à l'Ouest-Cameroun), le prix d'une bonbonne de 10 litres oscille entre 700 et 1500 voire 2000 francs CFA, une situation avantageuse aux producteurs ou vendeurs indépendants ayant choisi le " gré à gré ». Mais la situation est contraire d'avril à septembre (saison pluvieuse exclusivement) ${ }^{12}$ excepté le mois d'août (période de grandes manifestations culturelles : organisation des congrès et comités de développement des différents quartiers du village). Au cours de cette période, les prix pratiqués sont dérisoires et les produc-

12. Il faut tout de même noter que la situation connaît une nette évolution à cause de l'organisation des funérailles, un phénomène qui prend de plus en plus corps et s'intensifie dans le village Babadjou. teurs indépendants se retrouvent parfois avec des quantités invendues. Le contrat liant la vendeuse au producteur a l'avantage d'assurer à ce dernier l'écoulement de la totalité de sa production.

Dans l'ensemble, l'affluence constatée autour de la commercialisation du vin de raphia traduit la contribution monétaire que génère une « activité de cueillette » dans le budget familial dans un contexte de recomposition économique.

\section{Les impacts socio-économiques}

Bien qu'étant une activité de cueillette, la commercialisation du vin de raphia participe à la mobilisation d'un revenu non négligeable tant pour les producteurs, le coxeur, que pour les buyam-sellams. Au regard de l'investissement en travail et en capital limité en ce qui concerne les producteurs, les revenus monétaires générés par l'exploitation d'une raphiale sont absolument remarquables. À Babadjou, le bénéfice moyen par producteur/jour varie de 300 francs CFA pour le petit exploitant à 5000 francs CFA pour le gros exploitant, soit 9000 et 150000 francs CFA respectivement par mois. Dans d'autres régions tropicales la consommation du vin de raphia a connu une montée en puissance. À ce titre, Delarue (2007) précise qu'en région de Kpèlè (République de Guinée), la part du vin de raphia dans la valeur ajoutée au budget familiale chez les agriculteurs qui en produisent va de 15 à $69 \%$. Les revenus générés s'échelonnent de 1300000 GNF à 3700000 GNF (le litre est vendu entre 150 et $250 \mathrm{GNF}$ ) : pour obtenir de tels revenus en une année avec la production caféière, il faut investir au minimum de 260 à 740 jours de travail familial.

À Babadjou comme dans d'autres villages de l'Ouest-Cameroun où l'exploitation du raphia semble être considérable, ce produit ne s'intègre pas dans les circuits 


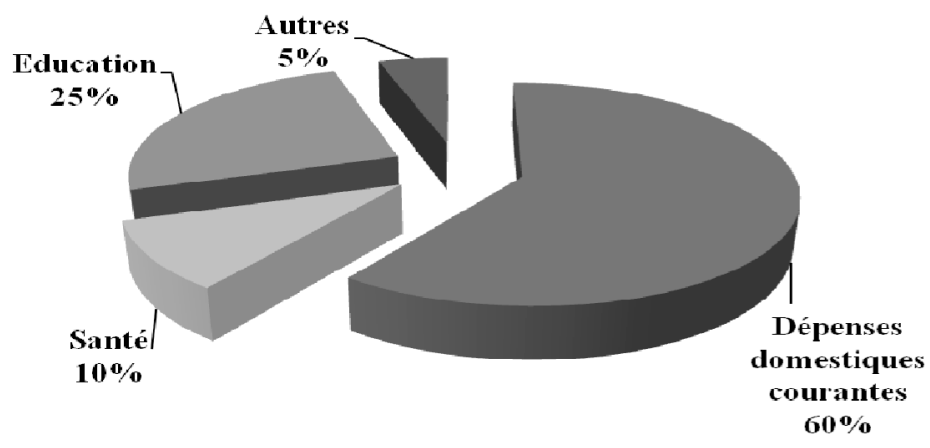

Source : I'auteur.

économiques structurés (car non imposable), mais son importance s'apprécie à travers la couverture quotidienne de multiples charges familiales (figure 7) puisque contrairement au café dont « l'argent finit vite puisqu'il est engagé par anticipation [...], la vente du vin de raphia offre au paysan, un revenu régulier, étalé sur l'année » (Gautier, Fadani, 1994) lui permettant d'honorer les engagements sociaux ou à couvrir les dépenses domestiques courantes telles que : pétrole, huile, savon, sel... Les producteurs utilisent les revenus issus de la vente du vin de raphia pour subvenir à leurs besoins de base et pour les réinvestir dans d'autres activités.

La quantité de charges familiales supportée par les revenus, le nombre de producteurs exclusifs ainsi que les quantités de vin écoulées par jour et par marché justifient le fait que le vin de raphia soit devenu une spéculation si importante dans l'économie des ménages de Babadjou qu'elle bouleverse non seulement les rapports sociaux mais les rapports à la terre. Les résultats d'une étude de rentabilité (tableau 2) menée par Gautier et Fadani en 1992 et qui demeurent d'actualité, présentent valablement la part du raphia dans l'économie des ménages. A priori, les revenus du raphia semblent aléatoires, car il faut attendre la maturité du pied (en moyenne, 7 à 10 ans voire plus après la plantation) et la production n'est prévisible ni en termes de date ni de durée (un pied peut produire du vin pendant deux semaines ou pendant trois mois). Aussi, les paysans qui ont de petites surfaces plantées en raphias peuvent passer parfois plusieurs mois sans aucun pied à récolter. Pour pallier cette difficulté, les producteurs prennent des raphiales en location auprès des veuves ou des personnes âgées incapables de s'y investir. Toutefois, les bénéfices dégagés par la vente du vin de raphia, restent sans commune mesure avec les revenus d'une activité caféière résiduelle. À la différence du café ou du maraîchage, la vente du vin de raphia offre, la possibilité d'engendrer un revenu régulier et permanent permettant aux paysans de mieux valoriser leurs revenus.

\section{Effets induits du développement de la filière vin de raphia}

\section{Spéculation foncière des parcelles à raphiales}

Aussi, à Babadjou, contrairement aux villages Bafou et Baleveng, les revenus tirés de la vente du vin de raphia ont donné aux 
Tableau 2. Rentabilité comparée d'un hectare de production de vin de raphia, de café arabica et de tomate (1991-1992)

\begin{tabular}{|c|c|c|c|c|}
\hline & \multicolumn{2}{|c|}{ Café arabica } & \multirow{2}{*}{ Raphia } & \multirow{2}{*}{ Tomate } \\
\hline & (1) & (2) & & \\
\hline $\mathrm{Nb}$ de pieds/ha (3) & 1600 & 1600 & 3000 & -- \\
\hline Production (3) & 4 & 4 & 10000 & 400 \\
\hline Prix CFA/unité & 24000 & 10000 & 25 & 800 \\
\hline Revenus bruts (CFA/ha) & 96000 & 40000 & 62500 & 320000 \\
\hline Engrais chimiques (CFA) & 6450 & 6450 & -- & 80000 \\
\hline Fumure (CFA) & 2500 & 2500 & -- & 35000 \\
\hline Produits phytosanitaires (CFA) & -- & -- & -- & 85000 \\
\hline Semence & -- & -- & -- & 30000 \\
\hline Dame-jeanne & -- & -- & 2400 & -- \\
\hline Coûts variables & 8950 & 8950 & 2400 & 230000 \\
\hline Marge brute (CFA) & 87050 & 31050 & 60100 & 90000 \\
\hline Heure travail/an/ha & 480 & 480 & 1095 & 1200 \\
\hline Marge brute/jour travail & 1088 & 388 & 329 & 450 \\
\hline Marge brute/CFA investissement & 13,55 & 4,81 & 25,04 & 1,3 \\
\hline
\end{tabular}

Note : (1) Avant 1989 ; (2) après 1989 ; (3) sacs de 60 kg pour le café, litre pour le vin de raphia ; sceau de 15 litres pour les tomates

Source : Gautier (1992). Modélisation basée sur les données de terrains recueillies par des enquêtes de suivi permanent sur 60 exploitations (Fadani, 1991-1992 : projet "Politiques des prix agricoles » financé par la CEE et par des enquêtes en profondeurs sur le fonctionnement des raphiales).

raphiales une plus-value. Insidieusement, le statut des raphiales est passé du social (don, legs...) à la spéculation. Ainsi, les « appétits » fonciers se sont aiguisés et les modes d'appropriation ont profondément évolué. Du droit d'exploiter jadis octroyé gratuitement par l'héritage ou par métayage $^{13}$, les conditions de location et de vente d'une parcelle dans les bas-fonds se sont renforcées à cause des avantages comparatifs là où ils existent (raphias, eau...). Par exemple, les prix de vente d'une parcelle à raphia ont connu une hausse considérable. Ainsi, une parcelle de $100 \mathrm{~m}^{2}$ qui se négociait à moins de 30000 francs CFA dans les années 1980 est de nos jours vendue autour de 300000 francs CFA. Dans

13. C'est un bail rural qui consiste à remettre au propriétaire soit le $1 / 3$ de la récolte, du vin à la demande, le versement de $60 \%$ des revenus de la vente. Il est pratiqué par des élites urbaines, des personnes âgées et des veuves qui ne disposent pas de temps et de force physique pour exploiter leurs plantations. cette transaction, seules des élites urbaines, de véritables « agriculteurs au col blanc », parviennent à les acquérir. Mais de plus en plus de jeunes ruraux aidés par leurs parents s'y investissent ou exploitent des parcelles héritées de leurs parents ou prises en location. Sur un tout autre plan et comparativement aux autres biens, comme le souligne Foko (1997), le palmier raphia est devenu au même titre que le café un élément de garantie matérielle le plus répandu. Contrairement au vivrier marchand, nous n'assistons pas à une profonde mobilité spatiale des raphiales.

\section{2. Émergence de " nouveaux riches »}

Dans la paysannerie en général, l'engouement pour les nouvelles spéculations marchandes et stratégies compensatrices en période post-crise de café a formé des catégories composites de l'évolution socioéconomique. Les jeunes déscolarisés et migrants de retour des villes, sans emplois, 
appauvris financièrement, et fragilisés socialement depuis la décennie 1990, constituent un groupe qui a su réinvestir, comme dans le vivrier marchand, la production et la commercialisation du vin de raphia avec pour objectif de trouver un revenu complémentaire pour une véritable insertion dans le secteur économique. Le cas de Roger K. est similaire à celui de nombreux jeunes. Déscolarisé, 32 ans, rentré à Babadjou en 2005, il a contracté un prêt à hauteur de 400000 FCFA qui lui a permis de prendre en location une raphiale de 100 pieds, vieille de 35 ans. Grâce à la vente du vin de raphia $^{14}$, il a pu entre 2005 et 2010 rembourser sa dette, s'offrir une case d'une valeur de 550000 francs CFA et une moto de marque KYMCO à 450000 francs CFA (TTC). Dès lors, l'augmentation de son capital lui a permis de s'investir dans la pratique du maraîchage. En dehors des producteurs, les revendeuses et les coxeurs réalisent désormais de gros bénéfices. L'analyse des données de terrain, a révélé qu'une vendeuse peut écouler en moyenne 100 litres/j à raison de 150 francs CFA le litre. Soit, une marge bénéficiaire brute de l'ordre de 7000 à 8000 francs CFA/jour. Grâce à cette activité principale, elle a un revenu mensuel évalué à environ 225000 francs CFA. Ce qui équivaudrait à une épargne d'environ 2700000 francs CFA par an même si les revendeuses de vin de raphia sont assujetties au paiement d'un impôt libératoire dont le montant s'élève à 11000 FCFA/an. En clair, ces exploitants avec ces revenus, contrairement aux anciens caféiculteurs, forment dans leur milieu respectif, la catégorie de « nouveaux riches ».

En conséquence, le revirement des jeunes désœuvrés à la vente du vin raphia et l'accumulation des capitaux contribuent à accélérer les changements sociaux : rapports de genre et intergénérationnels

14. Outre son statut de vigneron, il est devenu pluriactif : intermédiaire commercial de vin de raphia et moto taximen. comme mode de régulation se recomposent peu à peu. Désormais, les femmes du vivant de leur mari ou veuves s'investissent dans la plantation des raphiales dans les parcelles qu'elles cultivent à travers leurs enfants mâles, comme ce fut le cas pour la caféiculture. De plus en plus, le contrôle des raphiales est à l'origine des luttes réelles observées entre hommes et femmes, entre migrants de retour et certains dignitaires locaux dans de nombreuses familles polygamiques au cas où le chef de famille serait décédé. Des informations recueillies auprès des tribunaux de Mbouda, les luttes liées à l'exploitation des raphiales figurent parmi de nombreux griefs à l'origine des conflits fonciers à l'Ouest-Cameroun en général et à Babadjou en particulier.

Les anciens caféiculteurs face à l'engouement des stratégies compensatrices en période post-crise de café, forment le groupe composite des «perdants » de l'évolution socio-économique observée dans la campagne en général et autour du vin de raphia pour deux raisons : la pauvreté et le poids de l'âge (plus de 60 ans). Et ce d'autant plus que la production du vin bien que moins contraignante, nécessite de la force physique.

$$
\begin{aligned}
& * \\
& * *
\end{aligned}
$$

Face à la déprise caféière et à ses effets subséquents, les paysans ont développé un ensemble de stratégies pour s'assurer un revenu. Parmi ces nouvelles opportunités, le vin de raphia qui a fait l'objet de cette étude a non seulement contribué à valoriser davantage le capital terre et travail mais a trouvé des débouchés commerciaux. Le nombre de commerçantes toujours croissant dans les marchés ainsi que des coxeurs est un indicateur de taille du rôle que joue la vente du vin de raphia auprès des populations défavorisées. Nous pouvons ainsi comprendre que si le vin de raphia demeure socialement indispensable pour des populations (Babadjou) restées dans l'ensemble 
attachées aux valeurs culturelles, son commerce est économiquement important. Mais la pratique d'une agriculture marâ̂chère de contre-saison essentiellement par les jeunes et qui rapporte potentiellement bien à l'Ouest du Cameroun est entrée en concurrence avec la raphiale située en fond de vallée. Aussi, l'apprêté au gain pousse certains producteurs à fournir du vin de mauvaise qualité qui discrédite la filière.
Ainsi, comme gage de la durabilité et d'une valeur ajoutée substantielle pour l'économie dans les ménages, des mesures doivent être prises pour protéger la ressource. Ceci permettra de stabiliser le microclimat, de sécuriser la production et d'améliorer le fonctionnement de la filière vin de raphia afin de répondre durablement à la demande locale et surtout urbaine devenue de plus en plus croissante.

\section{RÉFÉRENCES BIBLIOGRAPHIQUES}

Bucrep (2005). Rapport de présentation des résultats définitifs du $3^{e}$ Recensement général de la population et de l'habitat du Cameroun. Yaoundé, $67 \mathrm{p}$.

Delarue J. (2007). Mise au point d'une méthode d'évaluation systémique d'impact des projets de développement agricole sur le revenu des producteurs. Étude de cas en région Kpèlè (République de Guinée). Thèse pour obtenir le grade de Docteur de l'Institut des Sciences et Industries du Vivant et de l'Environnement (Agro Paris Tech). Spécialité : Agriculture Comparée, Paris, 564 p.

Dongmo J.-L. (1976). Production et commercialisation des denrées alimentaires à destination des villes dans la province de l'ouest au Cameroun. In «Recherches sur l'approvisionnement des villes », Bordeaux, Centre d'étude de géographie tropicale du CNRS, coll. « La croissance urbaine dans les pays tropicaux », p. 47-74.

Dongmo J.-L. (1981). Le dynamisme bamiléké (Cameroun), La maîtrise de l'espace agraire. Yaoundé, CEPER, vol. 1, 424 p.

Fadani A. (1992). Farm surveys in coffee based farming systems in selected areas of the West, North-West and Littoral Provinces. Dschang/ Hohenheim, EEC-University of Hohenheim, Agricultural Price Policy Project.

Foko E. (1997). La pratique du prêt avec remise de gage : instrument de financement en milieu rural au Cameroun. Économie rurale, vol. 241, n 9 , p. 43-47.
Fongang Fouepe G.-H. (2008). Les mutations $d u$ secteur agricole bamiléké (Cameroun) étudiées à travers ses acteurs : une analyse à partir des localités de Fokoué et de Galim. Thèse de Doctorat de Sociologie, Paris, Institut des Sciences et Industries du Vivant et de l'Environnement (AgroParisTech).

Gautier D. (1992). Production et productivité d'un peuplement de Raphia mambillensis. Département de Foresterie, Centre Universitaire de Dschang, Cameroun.

Gautier D., Fadani A. (1994). Le raphia face à la crise : une production complémentaire qui pourrait être (re)valorisée. In G. Courade (dir.), Le village camerounais à l'heure de l'ajustement, Paris, Karthala, 410 p.

Guillermou Y., Kamga A. (2004). Les organisations paysannes dans l'Ouest-Cameroun. Palliatif à la crise ? Études rurales, vol. 1-2, $\mathrm{n}^{\circ} 169-170$, p. 61-76.

Guillermou Y. (2007). Organisations de producteurs et dynamiques paysannes dans l'OuestCameroun, Afrique contemporaine, vol. 2, $\mathrm{n}^{\circ} 222$, p. 251-271. Url : http://etudesrurales. revues.org/document5922.html

Huetz De Lemps A. (2001). Boissons et civilisations en Afrique. Presses universitaires de Bordeaux, Grappes et Millésimes.

Kaffo C. (1998). L'exploitation agropastorale des hautes pentes des monts Bamboutos, comme une alternative à la crise du café. Mémoire de Maîtrise de géographie, Université de Dschang, Cameroun, 98 p. 
Kaffo C. (2005). Cultures maraîchères dans les montagnes du Cameroun occidental. $\mathrm{Ca}$ hiers Agricultures, vol. 6, $\mathrm{n}^{\circ}$ 14, p. 517-24.

Kuété M., Uwizeyimana L. (2000). Déprise caféière et mutations socio-économiques sur les Hautes Terres de l'Ouest-Cameroun. GEODOC, $\mathrm{n}^{\circ} 51$, série MOCA n 8 « Montagnes et Café », Université de ToulouseLe- Mirail, France, 144 p.

Meutchieye F. (2007). Approvisionnement de la ville de Bafoussam en produits des raphiales : impacts économiques et environnementaux. Thèse de Master en Géographie, Université de Dschang, 130 p.

Moffo F. (2010). Rapport de stage de découverte du monde rural effectué à Babadjou du $1^{\text {er }}$ au 30 septembre 2010. École des sciences et de médecine vétérinaire (ESMV), Université de Ngaoundéré, Cameroun, 45 p.

Ngapgue J.-N. (2007). Le maraîchage et le vivrier marchand comme solution à la crise caféière dans la région de Foumbot. Thèse de Doctorat $(\mathrm{PhD})$ de Géographie, Université de Dschang, Cameroun, 597 p.

Nkankeu F., Kaffo C. (2012). Faillite de la caféiculture comme moteur du développement local à l'Ouest-Cameroun : leçons à tirer de trois décennies d'encadrement coopératif du monde rural (1960-1990). In Tsalefac M.,
Kamdem, P. « Les mutations sociospatiales au Cameroun, Mélanges en Hommage au Pr. Jean Louis Dongmo », Yaoundé, IRESMA, $327 \mathrm{p}$.

Oyono Ph.-R. (1997). Ruptures socio-économiques et surexploitation du palmier raphia par les populations forestières de Lomié, Sud-Est du Cameroun. Bulletin FTPP, p. 27-33.

Randrianjafy Zarasoa J.-N., Roger E. (2005). Fonctions économiques et écologiques du raphia, une plante à usage multiple. Faculté des Sciences, Université d'Antananarivo, Madagascar. URL : http://www.tanisiaina. com/ consulté le 15 juin 2011.

Seignobos Ch. (2002). Trente ans de bière de mil à Maroua du saré à Bil Bil au bistrot ? MEGA-TCHAD, p. 1-23.

Tsotezo Nitcheu C. (2001). Crise en milieu rural et stratégies de reconversion paysannes : cas de la localité de Babadjou dans le Bamboutos, Ouest-Cameroun. Mémoire de maîtrise de géographie, Université de Dschang, Cameroun, $116 \mathrm{p}$.

Uwizeyimana L. (2009). Après le café, le marâ̂chage ? Mutations des pratiques agricoles dans les Hautes Terres de l'Ouest-Cameroun. Les Cahiers d'Outre-Mer, $\mathrm{n}^{\circ}$ 247, URL : http://com.revues.org/index5675.html. 